\title{
Granulocyte colony-stimulating factor inhibits CXCR4/SDF-1 $\alpha$ signaling and overcomes stromal-mediated drug resistance in the HL-60 cell line
}

\author{
XIANFU SHENG, HUA ZHONG, HAIXIA WAN, JIHUA ZHONG and FANGYUAN CHEN
}

Department of Hematology, Ren Ji Hospital, School of Medicine, Shanghai Jiao Tong University, Shanghai 200127, P.R. China

Received February 5, 2015; Accepted March 22, 2016

DOI: $10.3892 /$ etm. 2016.3268

\begin{abstract}
Combining cytarabine, aclarubicin and granulocyte colony-stimulating factor (G-CSF) has demonstrated marked efficacy in the treatment of elderly and relapsed/refractory patients with acute myeloid leukemia (AML); however, the role of G-CSF remains poorly understood. The present study aimed to investigate the ability of G-CSF to overcome stromal-mediated drug resistance and the underlying molecular mechanism. Two types of co-culture models were established in the HS-5 human bone marrow/stromal and HL-60 human promyelocytic leukemia cell lines, in order to imitate the interactions between stromal and leukemia cells in vitro, which is mediated by the stromal cell-derived factor (SDF)- $1 \alpha$ signaling axis. In the present study, HL-60 cells were attracted and adhered to HS-5 cells using migration assay and flow cytometry, respectively; however, these interactions were inhibited by treatment with G-CSF and/or the C-X-C chemokine receptor type 4 (CXCR4) antagonist, AMD3100. Co-culture with HS-5 cells, including direct and indirect contact, protected HL-60 cells against spontaneous apoptosis or drug-induced apoptosis; however, these protective effects were disrupted by treatment with G-CSF and/or AMD3100. Notably, G-CSF and/or AMD3100 did not alter cell viability or apoptosis when HL-60 cells were cultured with medium alone. In addition, G-CSF significantly reduced the expression levels of surface CXCR4 protein, total CXCR4 protein and CXCR4 mRNA, and significantly upregulated the expression of microRNA (miR)-146a. Conversely, AMD3100 significantly reduced surface CXCR4 expression levels, but not the total CXCR4, CXCR4 mRNA or miR-146a expression levels. The results of the present study suggested that interfering with the CXCR4/SDF-1 $\alpha$ signaling axis via G-CSF inhibited
\end{abstract}

Correspondence to: Mr. Hua Zhong, Department of Hematology, Ren Ji Hospital, School of Medicine, Shanghai Jiao Tong University, 160 Pu Jian Road, Shanghai 200127, P.R. China

E-mail: zhh_lj@hotmail.com

Key words: drug resistance, microRNA-146a, AMD3100, granulocyte colony-stimulating factor, $\mathrm{C}-\mathrm{X}-\mathrm{C}$ chemokine receptor type $4 /$ stromal cell-derived factor- $1 \alpha$ axis the migration and adhesion of HL-60 cells to HS-5 cells and eliminated HS5 cell-mediated protective effects. Furthermore, G-CSF administration reduced CXCR4 expression levels by upregulating the expression of miR-146a, whereas AMD3100 appeared to be predominantly dependent on receptor internalization. Therefore, a G-CSF/miR-146a/CXCR4 pathway may explain how G-CSF inhibits CXCR4/SDF-1 $\alpha$ signaling and overcomes stromal cell-mediated drug resistance in acute myeloid leukemia.

\section{Introduction}

Acute myeloid leukemia (AML) remains the most common acute leukemia among adults (1). It is a heterogeneous hematological malignancy characterized by the clonal expansion of myeloid blasts in peripheral blood, bone marrow (BM), and/or other tissues. Despite the high response rate of AML to chemotherapy (the complete remission rate is $\sim 60-80 \%$ ) (2), the majority of patients with AML relapse, which has been attributed to residual disease in the BM (3). The establishment of separate regimens for elderly patients, relapsed/refractory patients or secondary AML patients (who are receiving cytotoxic therapy for solid tumors or hematologic malignancies) may attenuate the poor outcomes that are exhibited by these groups when treated with the standard cytarabine and an anthracycline. These poor outcomes may be due to drug resistance, comorbidity, Eastern Cooperative Oncology Group performance status or treatment-associated mortality $(4,5)$. The cytarabine, aclarubicin and granulocyte colony-stimulating factor (G-CSF) (CAG) treatment regimen was initially applied to these patients groups in Japan (6), and widespread popularization in Asia followed (7). Although the CAG regimen has demonstrated marked therapeutic efficacy (6), the definite mechanisms underlying the CAG protocol remain poorly understood.

Leukemia cells exhibit an enhanced proliferation rate and defective apoptosis, as compared with normal cells; however, they have been shown to undergo spontaneous apoptosis when cultured in vitro, thus suggesting that the microenvironment may have a significant protective role (8). Leukemia cells reside in BM niches, which are comprised of endothelial cells, perivascular reticular cells, osteoblasts, osteoclasts and stromal cells $(9,10)$; therefore, they are predominantly protected against spontaneous or drug-induced apoptosis (11). Interaction 
between leukemia cells and the BM microenvironment has been proposed as a potential mechanism for chemotherapy resistance, which is typically dependent on cell-to-cell contact and soluble signals, including cytokines, chemokines, growth factors and adhesion molecules $(12,13)$.

Chemokine stromal-derived factor- $1 \alpha(\mathrm{SDF}-1 \alpha)$, which is also known as $\mathrm{C}-\mathrm{X}-\mathrm{C}$ chemokine ligand 12, and its cognate receptor, $\mathrm{C}-\mathrm{X}-\mathrm{C}$ chemokine receptor type 4 (CXCR4), which is also known as cluster of differentiation (CD)184, are considered to be critical mediators of BM microenvironment/leukemic cell interactions (14-16). Binding of SDF-1 $\alpha$ induces conformational changes in CXCR4, resulting in its incorporation into lipid rafts and subsequent phosphorylation (17). Phosphorylation of CXCR4 activates cell signaling, which ultimately leads to alterations in gene transcription with consequent changes in the migration, adhesion, proliferation, survival and drug resistance of leukemia cells. There are two strategies for the modulation of CXCR4 expression. The first relies using a specific CXCR4 antagonist, such as Plerixafor (AMD3100), to compete with SDF-1 $\alpha$ for binding to CXCR4 $(18,19)$; and the second involves blocking CXCR4 expression at the mRNA level using small interfering microRNAs (miRs). miRs are small, non-coding RNAs (19-25 nucleotides) which modulate gene expression by targeting mRNA in a sequence-specific manner, leading to mRNA degradation or translational repression (20). Previous studies have suggested that miR-146a may have a pivotal role in downregulating CXCR4 expression $(21,22)$.

$\mathrm{G}-\mathrm{CSF}$, which is the main growth factor for the regulation of the proliferation and differentiation of myeloid cells, is a common agent used for mobilizing neutrophils, immature myeloid cells and hematopoietic stem cells (HSCs) from the $\mathrm{BM}$ into the peripheral blood. Previous studies have verified the role of G-CSF in CXCR4 downregulation $(23,24)$, and alterations in the expression levels of specific miRs, including miR-146a, have been reported during G-CSF-mediated mobilization of HSCs (25). The present study aimed to investigate the ability of G-CSF to inhibit CXCR4/SDF-1 $\alpha$ signaling, as well as the underlying molecular mechanisms, in order to overcome stromal cell-mediated drug resistance in AML.

\section{Materials and methods}

Cell culture. The HL-60 human acute promyelocytic leukemia cell line was a gift from the Institute of Hematology at the China Academy of Chinese Medical Sciences (Beijing, China). HL-60 cells were cultured in Iscove's modified Dulbecco's medium (IMDM; Gibco; Thermo Fisher Scientific, Inc., Waltham, MA, USA) supplemented with $20 \%$ fetal bovine serum (FBS; Gibco; Thermo Fisher Scientific, Inc.) at $37^{\circ} \mathrm{C}$ in a humidified incubator with $5 \% \mathrm{CO}_{2}$. The $\mathrm{HS}-5$ human $\mathrm{BM} /$ stromal cell line was purchased from ATCC (Manassas, VA, USA). HS-5 cells were cultured in Dulbecco's modified Eagle's medium (DMEM; Gibco; Thermo Fisher Scientific, Inc.) supplemented with $10 \%$ FBS in a humidified incubator at $37^{\circ} \mathrm{C}$ with $5 \% \mathrm{CO}_{2}$.

Reagents and antibodies. SDF-1 $\alpha$, AMD3100, recombinant human G-CSF, bovine serum albumin (BSA) and cytarabine were purchased from Sigma-Aldrich (St. Louis, MO, USA).
Phycoerythrin (PE)-conjugated mouse anti-human CD184 (clone 12G5; 561733), peridinin chlorophyll protein complex (PerCP)-conjugated anti-CD45 (clone 2D1; 561047) and PE-conjugated IgG2a isotypic control (555574) monoclonal antibodies (mAb) were purchased from BD Pharmingen (San Diego, CA, USA). Rabbit anti-human CXCR4 mAb (sc-9046) and horseradish peroxidase (HRP)-conjugated goat anti-rabbit immunoglobulin G (sc-2004) were purchased from Santa Cruz Biotechnology, Inc. (Dallas, TX, USA). Mouse anti-human $\beta$-tubulin mAb (KM9001T) was purchased from Sungene Biotech Co., Ltd. (Tianjin, China). $\beta$-actin mAB (P3002) was purchased from Abmart (Arlington, MA, USA).

Treatment of HL-60 cells. For functional assays, HL-60 cells were divided into four groups, as follows: i) Control group (medium alone); ii) G-CSF group (50 ng/ml G-CSF for $48 \mathrm{~h}$ ); iii) AMD3100 group (5 $\mu \mathrm{g} / \mathrm{ml} \mathrm{AMD3100} \mathrm{for} 30 \mathrm{~min}$ ); and iv) G-CSF plus AMD3100 group (initially cultured with $50 \mathrm{ng} / \mathrm{ml} \mathrm{G}-\mathrm{CSF}$ for $48 \mathrm{~h}$, then with $5 \mu \mathrm{g} / \mathrm{ml}$ AMD3100 for $30 \mathrm{~min})$. The group treated with AMD3100 only was used as a positive control.

Migration assay. Migration assays were performed using $6.5 \mathrm{~mm}$ Transwell assays with $8.0 \mu \mathrm{m}$ pore polycarbonate membrane inserts (Sigma-Aldrich). In order to increase the sensitivity of the cells to the SDF1- $\alpha$ chemoattractant and reduce non-specific migration caused by serum, HL-60 cells were starved for 24-48 $\mathrm{h}$ prior to performing the migration assays, and the FBS was replaced with $0.5 \%$ BSA. Briefly, the lower chamber was supplemented with $600 \mu \mathrm{l}$ IMDM medium containing $100 \mathrm{ng} / \mathrm{ml} \mathrm{SDF}-1 \alpha$ or $600 \mu \mathrm{l}$ HS-5 supernatant. Assay medium (IMDM with $0.5 \%$ BSA) without SDF-1 $\alpha$ was used as a baseline control. Subsequently, $5 \times 10^{5}$ cells in $100 \mu 1$ medium from all groups were added to the upper chamber and incubated for $3 \mathrm{~h}$ under $37^{\circ} \mathrm{C}$ and $5 \% \mathrm{CO}_{2}$ culture conditions. Following this, cells that had transmigrated into the lower chamber were collected and counted by flow cytometry (BD Pharmingen) at high flow for $20 \mathrm{sec}$. Control cells $\left(5 \times 10^{5}\right.$ in $600 \mu \mathrm{l}$ medium) were added to the lower chamber and counted under the same conditions. Results are shown as migration index $[$ mean \pm standard error of the mean (SEM)], which represents the ratio of the number of cells that transmigrated in the presence of chemoattractant and the number of cells that transmigrated in the absence of chemoattractant.

Adhesion assay. Firstly, a stromal cell layer composed of HS-5 cells was established by adding $1 \times 10^{5}$ cells/well to a 12 -well plate and culturing for $48 \mathrm{~h}$ until $80-90 \%$ confluence. Subsequently, HL-60 cells $\left(1 \times 10^{5}\right.$ cells/well) in the different groups were added to the HS-5 cell layer. Following co-culturing for $3 \mathrm{~h}$, non-adherent cells in the suspension were removed. Wells were washed three times with DMEM and adherent cells were detached by the addition of $0.5 \mathrm{ml} 0.25 \%$ trypsin-ethylenediaminetetraacetic acid (EDTA; Gibco; Thermo Fisher Scientific, Inc.) to each well. Subsequently, the suspension of the mixed cells group (HL60 and HS-5) was centrifuged at $1,400 \times \mathrm{g}$ at $4^{\circ} \mathrm{C}$ for $10 \mathrm{~min}$ and the supernatant was discarded. Cells in the pellet sediment were resuspended in $100 \mu \mathrm{l}$ DMEM and stained with $4 \mu \mathrm{l}$ PerCP-conjugated anti-CD45 mAb prior to counting by flow cytometry at high 
Table I. Primer sequences.

\begin{tabular}{lllc}
\hline Gene & \multicolumn{1}{c}{ Forward (5'-3') } & \multicolumn{1}{c}{ Reverse (5'-3') } & Product size (bp) \\
\hline h-ACTB & GGCACTCTTCCAGCCTTCC & GAGCCGCCGATCCACAC & 255 \\
CXCR4 & TCATCAGTCTGGACCGCTACC & GACGCCAACATAGACCACCTT & 95 \\
\hline
\end{tabular}

h-ACTB, human $\beta$-actin; CXCR4, C-X-C chemokine receptor type 4.

flow for $20 \mathrm{sec}$. An aliquot of the untreated cell population was counted under similar conditions as a control. Adhesion was calculated as a percentage of the number of initial cells (mean \pm SEM).

HS-5/HL-60 co-culture models and drug treatment. Firstly, a HS-5/HL-60 direct-contact co-culture model was established. Briefly, HS-5 cells (1x10 $10^{5}$ cells/well) were seeded onto 12-well plates two days prior to experiments and incubated at $37^{\circ} \mathrm{C}$ with $5 \% \mathrm{CO}_{2}$. Following confirmation of the confluence of the stromal layer by phase contrast microscopy (CX23; Olympus Corporation, Tokyo, Japan), HL- 60 cells ( $1 \times 10^{5}$ cells/well) from the various groups were added to HS-5 layers in the presence or absence of $0.05 \mu \mathrm{M}$ cytarabine. Following 48-h incubation, the cells were detached using $0.25 \%$ trypsin-EDTA, washed twice with phosphate-buffered saline (PBS) and resuspended in RPMI 1640 medium (Gibco; Thermo Fisher Scientific, Inc.) at a density of $5 \times 10^{5}$ cells $/ \mathrm{ml}$, since the manufacturer's protocol outlines that IMDM has been shown to influence optical density (OD) results.

Subsequently, the viability and apoptosis of the cells was assessed. For the viability assay, HS-5 cells ( $1 \times 10^{5}$ cells/well) were cultured in the absence of HL- 60 cells as baseline control. For the apoptosis assay, the cells were stained with PerCP-conjugated anti-CD45 mAb to separate HL-60 cells from HS-5 cells.

The present study also established a HS-5/HL-60 indirect-contact co-culture model. Briefly, HL-60 cells (1x $10^{5}$ cells/well) from the various groups were cultured with HS-5 supernatant or medium alone in the presence or absence of $0.05 \mu \mathrm{M}$ cytarabine for comparison. Following 48-h incubation, the cells were resuspended in RPMI 1640 medium at a density of $5 \times 10^{5}$ cells $/ \mathrm{ml}$, and viability and apoptosis assays were performed.

Viability and apoptosis assays. Cell viability was assessed using a Cell Counting kit-8 (CCK-8) assay (Dojindo Molecular Technologies, Inc., Kumamoto, Japan). Briefly, $100 \mu 1$ HL-60 cell suspension $\left(5 \times 10^{5} \mathrm{cells} / \mathrm{ml}\right)$ per well was seeded into a 96-well plate and $10 \mu \mathrm{l}$ CCK-8 solution was added to each well, followed by incubation under culture conditions for $4 \mathrm{~h}$. The absorbance of each well was determined at OD 490 and $630 \mathrm{~nm}$ using a Multiskan microplate reader (51119100; Thermo Fisher Scientific, Inc.). Results were expressed as percentages of control values.

Apoptosis was assessed by flow cytometry using a Alexa Fluor $^{\circledR} 488$ Annexin V Apoptosis kit (Invitrogen; Thermo Fisher Scientific, Inc.), according to the manufacturer's protocol. Briefly, HL-60 cells were washed with cold PBS and resuspended in $1 \mathrm{X}$ Annexin V-binding buffer at a density of $5 \times 10^{5}$ cells $/ \mathrm{ml}$. Subsequently, $5.0 \mu \mathrm{l}$ Alexa Fluor ${ }^{\circledR} 488$ Annexin $\mathrm{V}$ was added to $100 \mu \mathrm{l}$ cell suspensions, after which the samples were incubated at room temperature for $15 \mathrm{~min}$, followed by the addition of $400 \mu 1 \mathrm{lX}$ Annexin-binding buffer and gentle mixing. Samples were maintained on ice prior to analysis by flow cytometry. Percentage of apoptotic cells was calculated using the following formula: Apoptotic cells $(\%)=$ number of Annexin $\mathrm{V}^{+} \mathrm{HL}-60$ cells/total number of input cells x 100 .

Surface expression of CXCR4 on HL-60 cells. In order to evaluate CXCR4 surface expression, HL-60 cells $\left(1 \times 10^{6}\right.$ cells in $100 \mu \mathrm{l}$ medium) from the various groups were washed with PBS and labeled using 1.25 $\mu \mathrm{l}$ PE-conjugated anti-CXCR4. Subsequently, the cells were analyzed by flow cytometry using the FACSCalibur ${ }^{\mathrm{TM}}$ system (343020; BD Pharmingen) and Cell Quest 5.1 software (BD Pharmingen) for data acquisition and analysis. CXCR4 expression was assessed by comparison with cells incubated with $1.25 \mu \mathrm{l}$ PE-conjugated $\operatorname{IgG} 2 \mathrm{a}$ isotypic control antibody. Results are presented as the mean fluorescence intensity, which represents the ratio between the mean fluorescence values observed for the CXCR4-labeled cells and the cells labeled with the isotypic control.

Western blotting. HL-60 cells were lysed using radioimmunoprecipitation assay lysis buffer (Beyotime Institute of Biotechnology, Haimen, China) containing $50 \mathrm{mM}$ Tris (pH 7.4), 0.1\% sodium dodecyl sulfate (SDS) and $1 \mathrm{mM}$ phenylmethylsulfonyl fluoride protease inhibitor. Lysates were maintained on ice for $30 \mathrm{~min}$ following centrifugation at $14,000 \mathrm{x} \mathrm{g}$ for $3 \mathrm{~min}$ at $4^{\circ} \mathrm{C}$. Protein concentration of the supernatant was determined using a Bicinchoninic Acid Protein Assay kit (Beyotime Institute of Biotechnology), according to the manufacturer's protocol. Total protein $(30 \mu \mathrm{g})$ from each sample was separated by $10 \%$ SDS-polyacrylamide gel electrophoresis and transferred to polyvinylidene difluoride membranes (EMD Millipore, Billerica, MA, USA). Membranes were blocked with 5\% non-fat milk in Tris-buffered saline containing Tween-20 (TBST; Sigma-Aldrich) at room temperature for $30 \mathrm{~min}$, after which the membranes were incubated overnight at $4^{\circ} \mathrm{C}$ with anti-CXCR4 (1:250) and $\beta$-tubulin $(1: 3,000)$ primary antibodies. Following rinsing three times with $20 \mathrm{ml}$ TBST for $5 \mathrm{~min}$, the membranes were incubated with HRP-conjugated goat anti-rabbit immunoglobulin G secondary antibody $(1: 1,500)$ for $1 \mathrm{~h}$ at room temperature. Bands were detected by Western lightning enhanced chemiluminescence reagent (PerkinElmer, Inc., Waltham, MA, USA). Results were analyzed on a Tanon 5500 Chemiluminescence 
Imaging system (Tanon Science and Technology Co., Ltd., Shanghai, China) using ImageCal 4.0 software (Tanon Science and Technology Co., Ltd.) and were normalized to $\beta$-tubulin $(1: 3,000)$.

Reverse transcription-quantitative polymerase chain reaction (RT-qPCR) analysis. Total RNA was extracted from HL-60 cells using TRIzol reagent (Takara Biotechnology Co., Ltd., Dalian, China), according to the manufacturer's protocols. After DNase I treatment (Thermo Fisher Scientific, Inc.), cDNA was synthesized using RevertAid Reverse Transcriptase (Thermo Fisher Scientific, Inc.). PCR was performed using SYBR Green qPCR Mix (Dongsheng Biotech Co., Ltd., Guangzhou, China) on a Bio-Rad iQ5 PCR thermal cycler (Bio-Rad Laboratories, Inc., Hercules, CA, USA). Primers are shown in Table I, with the exception of the primer sequences for miR-146a, which were unavailable. qPCR was performed using a reaction volume of $20 \mu \mathrm{l}$ containing $10 \mu \mathrm{l}$ SYBR Green (2X), $1 \mu \mathrm{l} 0.5 \mu \mathrm{g} / \mu \mathrm{l}$ primers, $1 \mu 1 \mathrm{cDNA}$ template and $8 \mu 1$ water. PCR conditions were as follows: Denaturation at $95^{\circ} \mathrm{C}$ for $2 \mathrm{~min}$, followed by 40 cycles of denaturation at $95^{\circ} \mathrm{C}$ for $15 \mathrm{sec}$, annealing at $60^{\circ} \mathrm{C}$ for $20 \mathrm{sec}$ and extension at $72^{\circ} \mathrm{C}$ for $20 \mathrm{sec}$, with final elongation at $72^{\circ} \mathrm{C}$ for $5 \mathrm{~min}$. Each gene was tested and the respective melting curves were detected. All samples were measured in triplicate. Expression levels of CXCR4 and miR-146a were normalized to human $\beta$-actin and RNU6A reference gene levels, respectively. Mean $\mathrm{Cq}$ values from each sample were normalized against the mean $\mathrm{Cq}$ value of the reference genes. Relative expression levels were acquired using the $2^{-\Delta \Delta C q}$ method (26).

Statistical analysis. Data are presented as the mean \pm SEM of at least three independent experiments. Comparisons were performed using a two-tailed, unpaired Student's t-test. $\mathrm{P}<0.05$ was considered to indicate a statistically significant difference.

\section{Results}

$G$-CSF reduces the migration of $H L-60$ cells in response to SDF-1 $\alpha$ or HS-5 supernatant. Initially, the potential effect of G-CSF on the SDF- $1 \alpha$-induced migration of HL- 60 cells was examined in vitro. AMD3100-only treated HL-60 cells were used as a positive control and assay medium-only treated cells were used as the baseline. The migration index was markedly increased upon exposure of the control HL-60 cells to medium containing $100 \mathrm{ng} / \mathrm{ml} \mathrm{SDF-1} \alpha$, as compared with HL-60 cells exposed to assay medium without SDF- $1 \alpha(1.75 \pm 0.08$ vs. $1.00 \pm 0.02 ; \mathrm{P}<0.01)$. However, G-CSF and AMD3100 were demonstrated to significantly reduce the SDF-1 $\alpha$-induced migration of HL-60 cells, as compared with the control (1.27 \pm 0.01 and $0.86 \pm 0.06$ vs. $1.75 \pm 0.08$, respectively; $\mathrm{P}<0.01$; Fig. 1A). In addition, combination treatment with G-CSF and AMD3100 reduced the migration index to the greatest extent, as compared with either agent alone $(0.58 \pm 0.06$ vs. $1.27 \pm 0.01$ or $0.86 \pm 0.06 ; \mathrm{P}<0.01$; Fig. $1 \mathrm{~A})$. These results suggested that G-CSF may reduce functional CXCR4 expression and specific responses to SDF-1 $\alpha$ in HL-60 cells, which is similar to AMD3100 treatment.
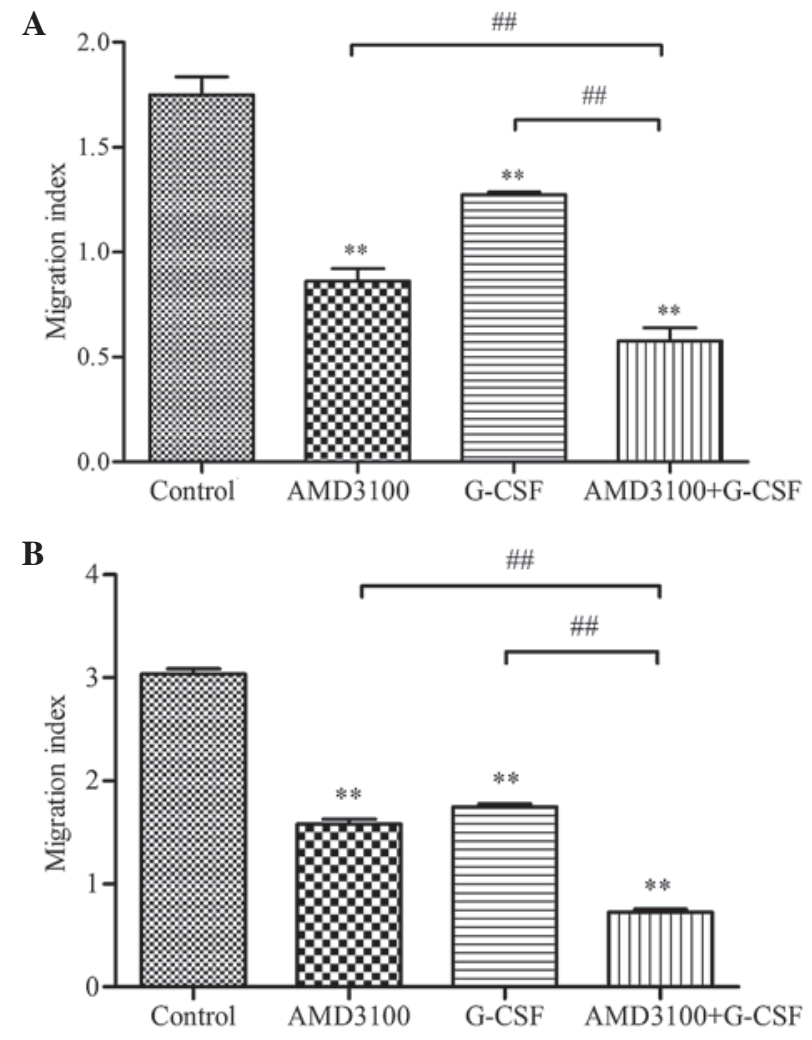

Figure 1. Effects of G-CSF and/or AMD3100 on SDF-1 $\alpha$ - or HS-5 supernatant-induced HL-60 cell migration. (A) HL-60 cells were tested for transwell migration in response to SDF-1 $\alpha(100 \mathrm{ng} / \mathrm{ml})$. (B) HL-60 cells were tested for trans-well migration in response to HS-5 supernatant. Data are presented as the mean \pm standard error of the mean of three independent experiments. ${ }^{* *} \mathrm{P}<0.01$ vs. the control group; ${ }^{\# \#} \mathrm{P}<0.01$ vs. the G-CSF plus AMD3100 group. G-CSF, granulocyte colony-stimulating factor; SDF-1 $\alpha$, stromal cell-derived factor-1 $\alpha$.

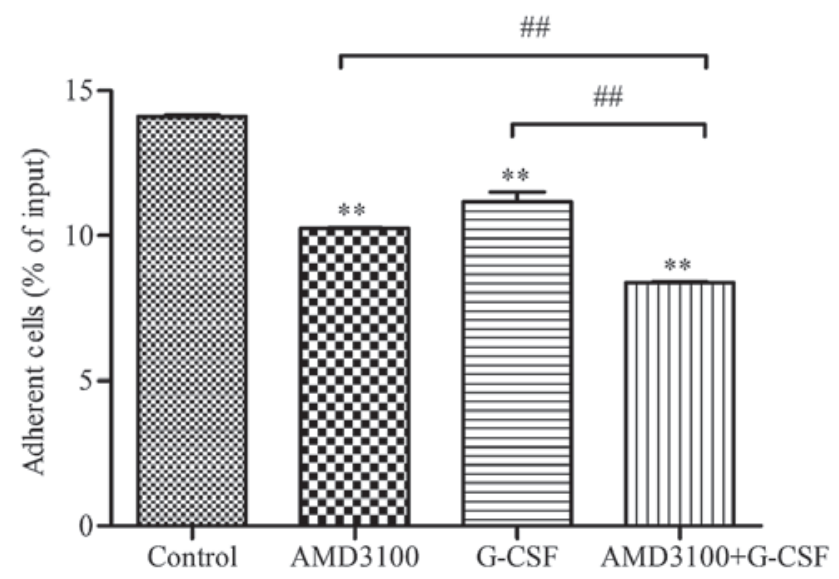

Figure 2. Effects of G-CSF and/or AMD3100 on HL-60 cell adhesion to HS-5 cells. Adherent cells were calculated as a percentage of input cells by flow cytometry. Data are presented as the mean \pm standard error of the mean of three independent experiments. ${ }^{* *} \mathrm{P}<0.01$ vs. the control group; ${ }^{\# \#} \mathrm{P}<0.05$ vs. the G-CSF plus AMD3100 group. G-CSF, granulocyte colony-stimulating factor.

The ability of HS-5 cells to secrete SDF1- $\alpha$ and attract HL-60 cells was evaluated using the HS-5 supernatant, rather than SDF- $1 \alpha$-containing medium, as a chemoattractant. Similar results were observed as when the SDF1- $\alpha$ medium 

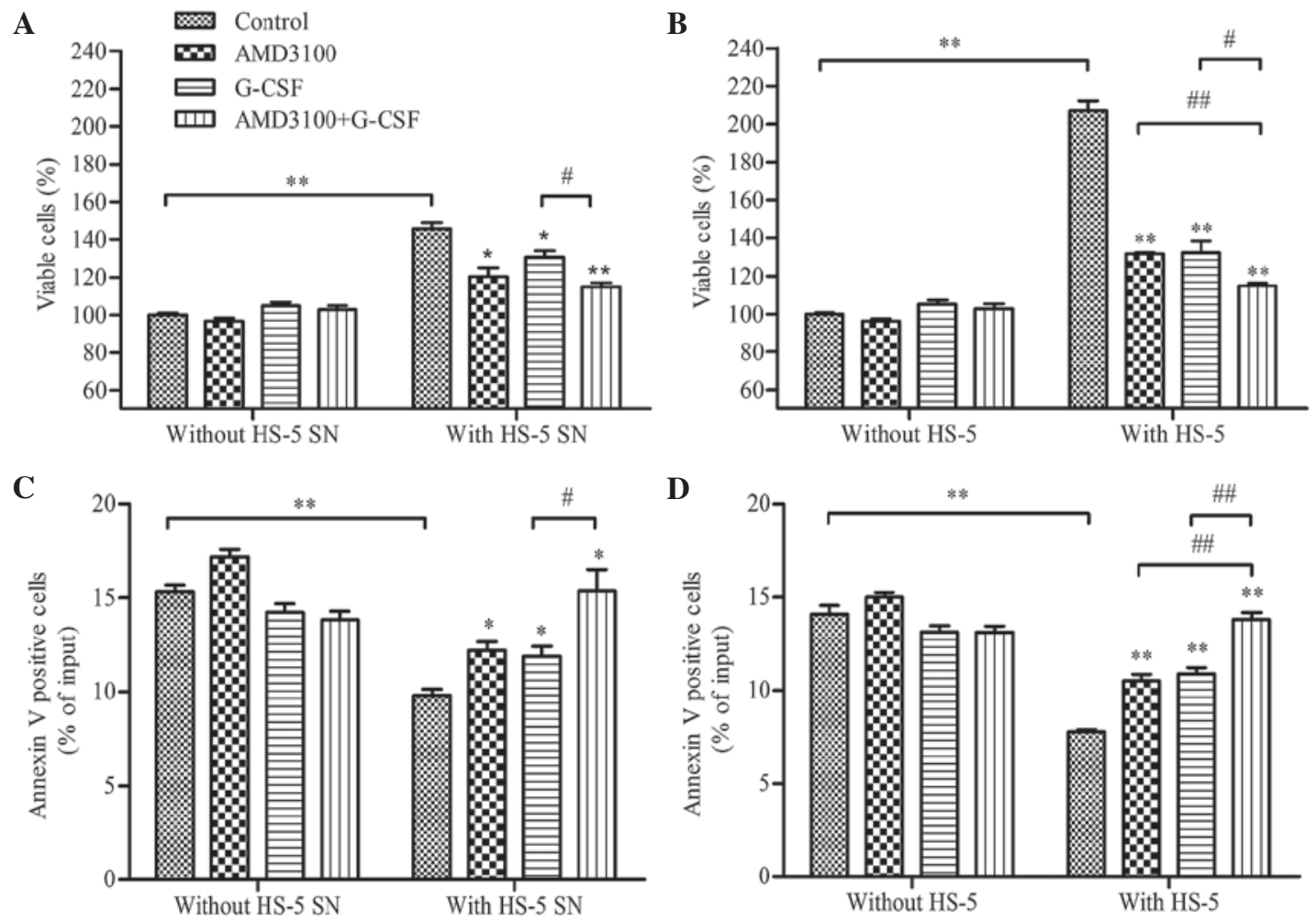

Figure 3. G-CSF and/or AMD3100 sensitize HL-60 cells to spontaneous apoptosis in HS-5/HL-60 co-culture models. HL-60 cells were co-cultured with (A and B) HS-5 supernatant, (C and D) HS-5 cells or medium only (control). (A and C) Cell viability (\%) was obtained following normalization of the results to the control group. (B and D) Cell apoptosis is shown as the percentage of Annexin $\mathrm{V}^{+}$cells (including early and late apoptosis). Data are presented as the mean \pm standard error of the mean of three independent experiments. ${ }^{*} \mathrm{P}<0.05$ and ${ }^{* *} \mathrm{P}<0.01$ vs. the control group; ${ }^{\#} \mathrm{P}<0.05$ and ${ }^{\# \#} \mathrm{P}<0.01$ vs. the G-CSF plus AMD3100 group. SN, supernatant; G-CSF, granulocyte colony-stimulating factor.

was used as the chemoattractant. The migration index of the control HL-60 cells exposed to HS-5 supernatant was markedly increased, as compared with the cells exposed to medium alone (3.04 \pm 0.05 vs. $1.00 \pm 0.04 ; \mathrm{P}<0.01$; Fig. 1B). Conversely, G-CSF and AMD3100 were demonstrated to significantly reduce the migration index of HL-60 cells, as compared with the control $(1.75 \pm 0.03$ and $1.58 \pm 0.05$ vs. $3.04 \pm 0.05$, respectively; $\mathrm{P}<0.01$; Fig. 1B). In addition, combination treatment with G-CSF and AMD3100 reduced the migration index to the greatest extent, as compared with either agent alone $(0.73 \pm 0.03$ vs. $1.58 \pm 0.05$ or $1.75 \pm 0.03$; P $<0.01$; Fig. $1 B)$. These data indicated that HS- 5 cells may produce SDF-1 $\alpha$ to attract HL-60 cells that express CXCR4 on their surface; whereas G-CSF may reduce surface CXCR4 expression levels synergistically with AMD3100.

$G$-CSF reduces the adhesion of HL-60 cells to HS-5 cells. Since drug resistance is predominantly dependent on cell-to-cell contact, the present study investigated the potential effect of G-CSF and/or AMD3100 treatment on the adhesion of HL-60 cells to HS-5 cells. The percentage of HL-60 cells that specifically adhered to the HS-5 cell layer was significantly reduced in the G-CSF (10.25 \pm 0.03$)$, AMD3100 $(11.17 \pm 0.35)$ and G-CSF plus AMD3100 $(8.39 \pm 0.03)$ groups, as compared with the control group (14.1 $\pm 0.06 ; \mathrm{P}<0.01$; Fig. 2). Furthermore, the greatest inhibition of adhesion was detected in the G-CSF plus AMD3100 group $(8.39 \pm 0.03)$, as compared with the G-CSF $(10.25 \pm 0.03)$ and AMD3100 $(11.17 \pm 0.35)$ groups $(\mathrm{P}<0.05$;
Fig. 2). These results suggest that HL-60 cells are attracted and adhere to HS-5 cells, and that G-CSF and AMD3100 are able to block these effects independently and synergistically.

HS-5 cells protect HL-60 cells from spontaneous apoptosis, whereas $G$-CSF reduces cell viability and restores apoptosis. In order to investigate the effect of G-CSF on HL-60 cells in diverse microenvironments, HL-60 cells were co-cultured with HS-5 supernatant (indirect contact), HS-5 cells (direct contact) or medium alone (control), following pretreatment with AMD3100, G-CSF, G-CSF plus AMD3100 or medium (control), and cell viability and apoptosis were analyzed after $48 \mathrm{~h}$. A protective effect was observed in the HL-60 cells co-cultured with the HS-5 supernatant; cell viability was significantly increased $(\mathrm{P}<0.01 ;$ Fig. $3 \mathrm{~A})$, and apoptosis was significantly reduced $(\mathrm{P}<0.01$; Fig. $3 \mathrm{~B})$, as compared with the HL-60 cells co-cultured with medium only. Notably, G-CSF and/or AMD3100 did not significantly effect the viability (Fig. 3A) or apoptosis (Fig. 3B) of HL-60 cells that had been cultured with medium alone (without HS-5 supernatant or HS-5 cells). However, pretreatment with G-CSF and/or AMD3100 significantly reduced the viability $(\mathrm{P}<0.05)$ and increased the apoptosis $(\mathrm{P}<0.05)$ of HL-60 cells co-cultured with HS-5 supernatant, with G-CSF plus AMD3100 demonstrating greater effects on HL-60 cell viability and apoptosis than either agent alone ( $\mathrm{P}<0.05$; Figs. $3 \mathrm{~A}$ and $\mathrm{B})$. These results suggested that G-CSF and AMD3100 antagonize the protective effect of HS-5 cells on HL-60 cells that become more 

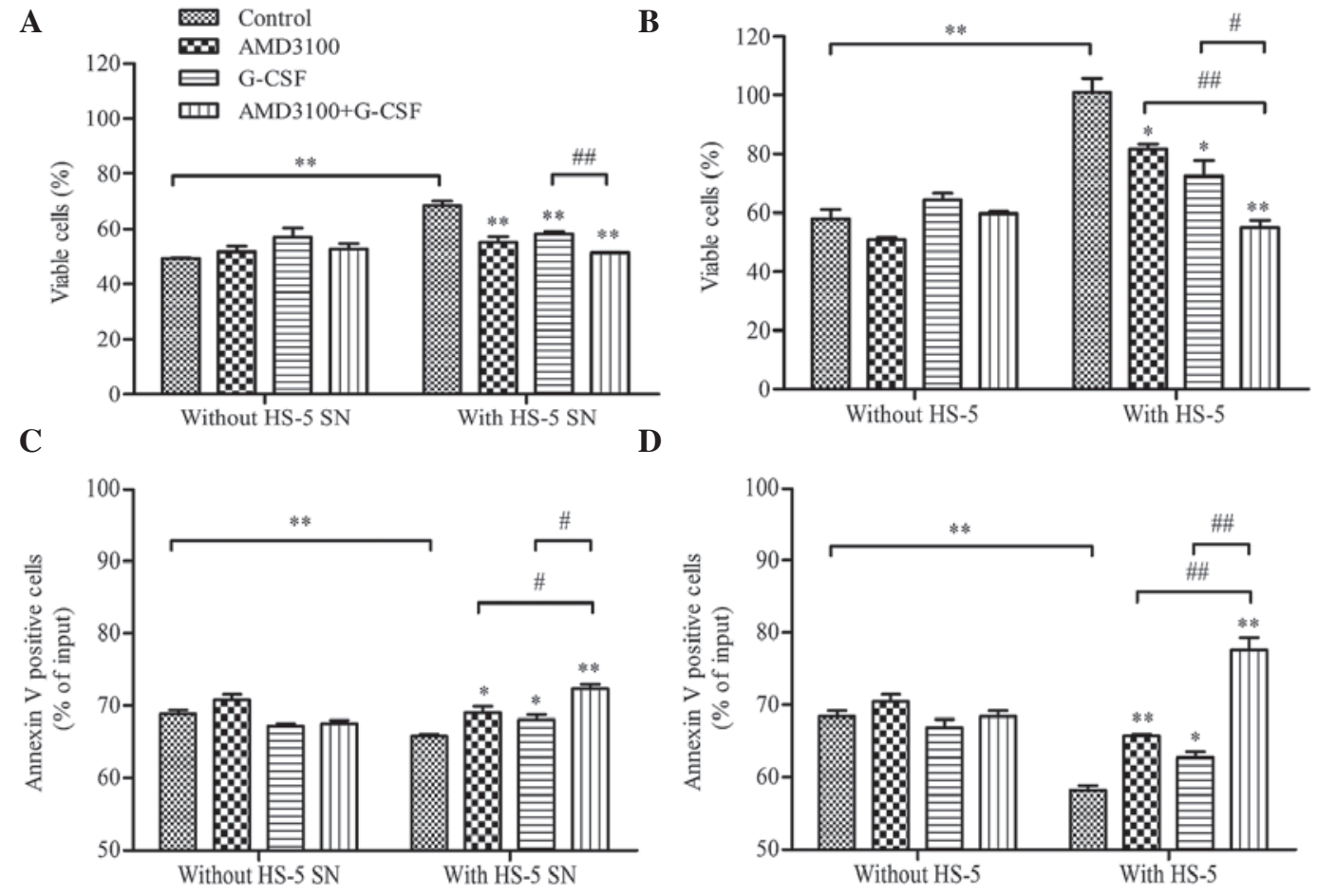

Figure 4. G-CSF and/or AMD3100 sensitize HL-60 cells to drug-induced apoptosis in HS-5/HL-60 co-culture models. HL-60 cells were co-cultured with (A and B) HS-5 supernatant, (C and D) HS-5 cells or medium only (control) in the presence of cytarabine. (A and C) Cell viability (\%) was obtained following normalization of the results to the control group. (B and D) Cell apoptosis was shown as a percentage of Annexin $\mathrm{V}^{+}$-cells (including early and late apoptosis). Data are presented as the mean \pm standard error of the mean of three independent experiments. ${ }^{*} \mathrm{P}<0.05$ and ${ }^{* * *} \mathrm{P}<0.01$ vs. the control group; ${ }^{\#} \mathrm{P}<0.05$ and ${ }^{\# \#} \mathrm{P}<0.01$ vs. the G-CSF plus AMD3100 group. SN, supernatant. G-CSF, granulocyte colony-stimulating factor.

sensitive to apoptosis, which may be rely on interfering with the CXCR4/SDF-1 $\alpha$ axis.

Similar results were observed for the HL-60 cells co-cultured with HS-5 cells (Figs. 3C and D). However, cell viability was significantly enhanced $(206.98 \pm 9.62 \%$ vs. $145.51 \pm 6.30 \% ; \mathrm{P}<0.01)$ and apoptosis was significantly reduced $(7.79 \pm 0.10 \%$ vs. $9.80 \pm 0.60 \% ; \mathrm{P}<0.01)$ for the HL-60 cells co-cultured with HS-5 cells, as compared with those co-cultured with HS-5 supernatant (Figs. 3A and B).

G-CSF sensitizes HL-60 cells to drug-induced apoptosis in HL-60/HS-5 co-culture models. The effect of G-CSF on the drug-induced apoptosis of HL-60 cells was evaluated using HL-60/HS-5 co-culture models. HL-60 cells were pre-treated with G-CSF, AMD3100, G-CSF plus AMD3100 or medium (control), and then co-cultured with HS-5 supernatant, HS-5 cells or medium in the presence of cytarabine $(0.05 \mu \mathrm{M})$. Following 48-h incubation, cell viability and apoptosis were analyzed. G-CSF and AMD3100 did not influence cell viability or apoptosis when HL-60 cells were co-cultured with medium alone in the presence of cytarabine (Fig. 4). As compared with the medium alone, co-culture with HS-5 supernatant or HS-5 cells protected HL-60 cells against cytarabine-induced apoptosis; cell viability was significantly increased $(\mathrm{P}<0.01$; Figs. 4A and $\mathrm{C}$, respectively) and apoptosis was significantly reduced ( $\mathrm{P}<0.01$, Figs. $4 \mathrm{~B}$ and $\mathrm{D}$, respectively). However, pretreatment with G-CSF and/or AMD3100 significantly inhibited the protective effects of the HS-5 supernatant or HS-5 cells, with G-CSF plus AMD3100 exhibiting a greater effect on HL-60 cell viability and apoptosis than either agent when used alone ( $\mathrm{P}<0.05$; Fig. 4). In addition, a more pronounced protective effect against cytarabine-induced apoptosis was observed for the HL-60 cells co-cultured with HS-5 cells, as compared with the HS-5 supernatant (cell viability: $101.04 \pm 4.72 \%$ vs. $68.31 \pm 1.71 \%$, $\mathrm{P}<0.01$; apoptosis: $58.20 \pm 0.63 \%$ vs. $65.75 \pm 0.33 \%, \mathrm{P}<0.05$; Fig. 4). These results suggest that the HS-5 supernatant and HS-5 cells exert pro-proliferative and anti-apoptosis effects, which may be increased when the leukemia cells are in physical contact with the stromal cells. Furthermore, the results suggested that G-CSF and AMD3100 may be able to restrict the stromal-based microenvironment-mediated protective effects on leukemia cells.

$G-C S F$ reduces the expression levels of CXCR4 $\mathrm{mRNA}$, surface protein and total protein, and increases the expression of miR-146a in HL-60 cells. In functional assays, G-CSF and AMD3100 were able to significantly inhibit the migration and adhesion of HL-60 cells, and the protective effects of stromal cells. In order to elucidate the association between the CXCR4/SDF-1 $\alpha$ axis and the stromal-mediated protective effects, the ability of G-CSF to alter the expression levels of CXCR4 was evaluated. Firstly, surface CXCR4 expression was evaluated using a PE-conjugated anti-CXCR4 monoclonal antibody and fluorescence-activated cell sorting. Mean fluorescence intensity ratios of cells pre-treated with G-CSF (9.87 \pm 0.45$)$, AMD3100 (7.89 \pm 0.13$)$ or G-CSF plus AMD3100 $(2.88 \pm 0.17)$ were significantly reduced, as compared with the control group $(14.29 \pm 0.32 ; \mathrm{P}<0.01)$. Furthermore, combined treatment with G-CSF and AMD3100 resulted in a greater reduction than when either agent was used alone $(\mathrm{P}<0.01$; 
A

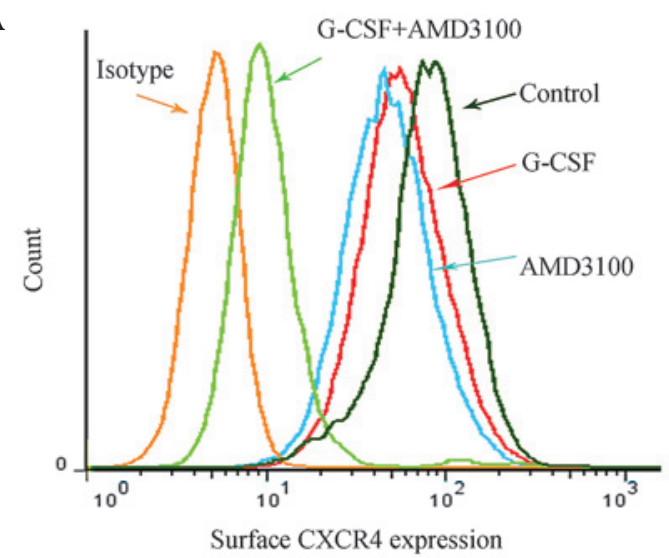

B

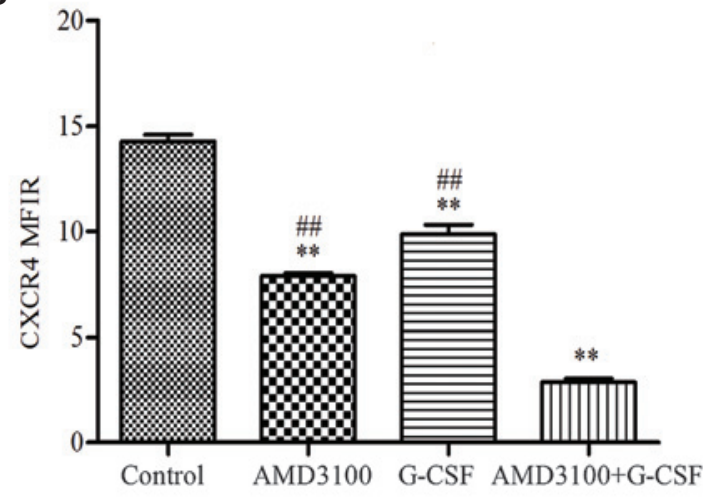

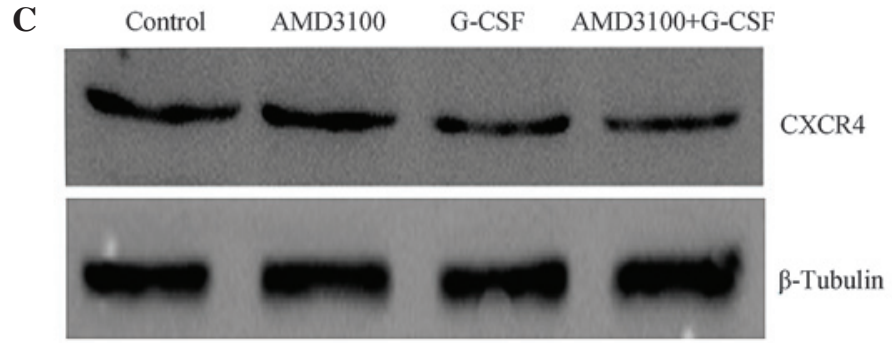

D

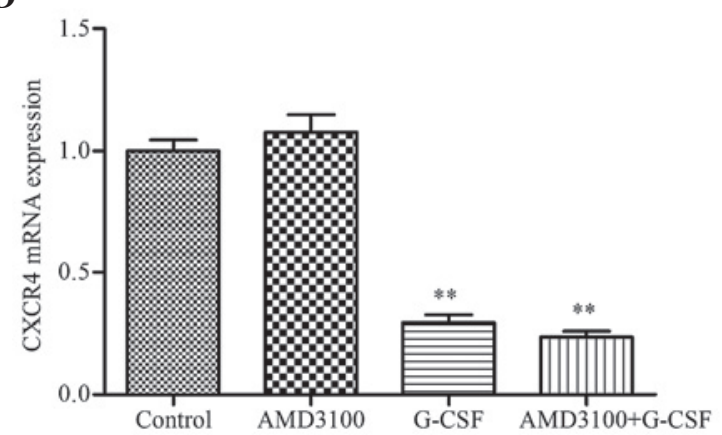

$\mathbf{E}$

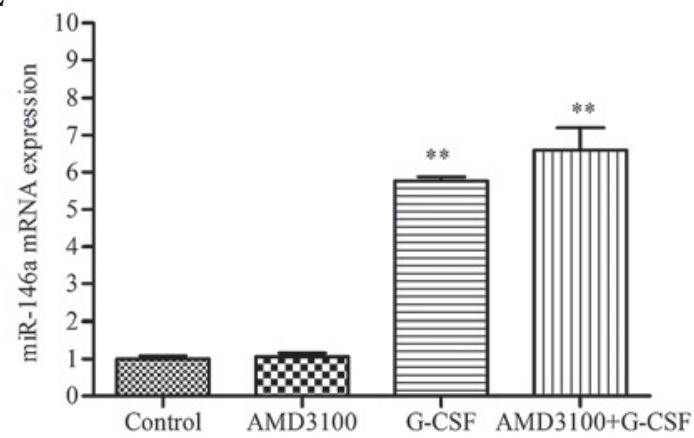

Figure 5. Effects of G-CSF and/or AMD3100 on the expression levels of (A and B) surface CXCR4 protein, (C) total CXCR4 protein, (D) CXCR4 mRNA and (E) miR-146a. (A) Cells were stained with phycoerythrin-conjugated anti-CXCR4 monoclonal antibody and surface CXCR4 expression was analyzed by flow cytometry. (B) MFIR quantitative analysis, representing the CXCR4 signal/isotypic signal ratio, was performed. (C) Total CXCR4 protein expression levels were measured by western blotting with specific antibodies against CXCR4. (D) CXCR4 mRNA and (E) miR-146a levels were analyzed by reverse transcription-quantitative polymerase chain reaction. Data are presented as the mean \pm standard error of the mean of three independent experiments. ${ }^{\# \#} \mathrm{P}<0.01$ vs. the AMD3100 + G-CSF group; ${ }^{* *} \mathrm{P}<0.01$ vs. the . G-CSF, granulocyte colony-stimulating factor; CXCR4, C-X-C chemokine receptor type 4; miR, microRNA; MFIR, mean fluorescence intensity ratio.

Fig. 5A). Next, the effect of G-CSF on the total CXCR4 content of HL-60 cells was assessed by western blotting, since CXCR4 is predominantly an intracellular protein (27). There was a marked reduction of the 39-kDa CXCR4-associated band in the HL-60 cells pretreated with G-CSF or G-CSF plus AMD3100, as compared with the control group, whereas AMD3100 did not affect CXCR4 protein expression (Fig. 5B). Subsequently, the expression levels of CXCR4 mRNA and miR-146a were analyzed by RT-qPCR. G-CSF significantly reduced CXCR4 mRNA expression $(\mathrm{P}<0.01)$ and increased miR-146a expression $(\mathrm{P}<0.01)$, as compared with the control; whereas AMD3100 treatment had no significant effect (Figs. 5C and D). In addition, a highly inverse correlation was observed between the expression levels of CXCR4 mRNA and miR-146a, which is indicative of post-transcriptional inhibition of gene expression.
Overall, these results suggest that G-CSF inhibits HL-60 cell migration and adhesion to HS-5 cells, and sensitizes them to spontaneous or drug-induced apoptosis by disturbing the CXCR4/SDF-1 $\alpha$ axis via miR-146a upregulation.

\section{Discussion}

Substantial progress in the treatment of AML has been achieved; in particular, the CAG regimen has demonstrated great success in the treatment of elderly, relapsed/refractory and secondary AML patients $(1,28)$. Previous in vitro and in vivo studies have highlighted the importance of G-CSF (29-31), demonstrating that G-CSF has a key role in the CAG regimen via cell cycle synchronization and cell mobilization. We suggest a novel hypothesis that G-CSF may weaken the protection of stromal cells and promote the apoptosis of leukemia cells. To the best 
of our knowledge, no direct evidence in support of this has been reported.

Spinello et al (32) demonstrated that patients with M4/M5 subtypes of AML had the highest levels of CXCR4 protein expression, those with M3 had the second highest, and those with M1/M2 had the lowest. Therefore, the present study selected a common human leukemia cell line, HL-60, which is thought to be a cell line of the AML-M2 subtype, and co-cultured it with the HS-5 human BM/stromal cell line to imitate the interactions between stromal cells and leukemia cells in vitro (33). AMD3100, which is a CXCR4 inhibitor, has been used in humans for $>10$ years as a HSC mobilizing agent (34). Previous studies have demonstrated that AMD3100 exerts an inhibitory effect on the CXCR4/SDF-1 $\alpha$ axis $(12,15,21,35)$; thus AMD3100 was used as a positive control in the present study.

Homing and retention in the BM are key protective mechanisms for cells to escape drug-induced apoptosis and are predominantly dependent on the CXCR4/SDF-1 $\alpha$ axis (14). Therefore, the present study investigated the effect of G-CSF on cell migration and adhesion, which partially reflect homing and retention, respectively. The results demonstrated that G-CSF significantly decreased the migration and adhesion of HL-60 cells to HS-5 cells, which was consistent with a previous study, in which a similar inhibitory effect was reported for AMD3100 (35). In addition, the present study demonstrated that G-CSF and AMD3100 had a greater inhibitory effect on cell migration than on cell adhesion, which may be due to the fact that cell adhesion involves numerous adhesion molecules, whereas cell migration is predominantly dependent on the CXCR4/SDF-1 $\alpha$ axis (36). Although cell adhesion in this assay did not only reflect CXCR4/SDF-1 $\alpha$ interactions, but also was dependent on contributions from other molecules induced by CXCR4 activation, these results still provide evidence that G-CSF may reduce functional CXCR4 levels in myeloid cells.

Viability and apoptosis assays performed in the present study demonstrated that co-culture with HS-5 supernatant and HS-5 cells was able to protected HL- 60 cells against spontaneous or drug-induced apoptosis. Notably, a greater protective effect was observed when HL-60 cells were co-cultured with HS -5 cells (direct contact), as compared with when they were co-cultured with HS-5 supernatant (indirect contact). These results suggested that the protective effects of stromal cells were predominantly dependent on physical contact, although soluble factors were also involved. Furthermore, G-CSF decreased the viability and promoted the apoptosis of HL-60 cells in the presence or absence of cytarabine, although it was unable to affect the viability and apoptosis of HL-60 cells cultured with medium alone. Similar results were observed for AMD3100. These results suggested that G-CSF and AMD3100 affected the survival and apoptosis of HL-60 cells by disrupting the interactions between HL-60 and HS-5 cells, potentially via the CXCR4/SDF-1 $\alpha$ axis, not as a result of their toxicity. In addition, to the best of our knowledge, the present study is the first to report synergistic effects for G-CSF and AMD3100 on cell migration, adhesion, survival and apoptosis in vitro, although previous studies have demonstrated their synergistic effect in the mobilization of HSCs $(37,38)$.

In functional assays, the present study demonstrated that G-CSF was able to disrupt HS-5/HL-60 cell cross-talk by interfering with the migration and adhesion of HL-60 cells to HS-5 cells, potentially via inhibition of the CXCR4/SDF-1 $\alpha$ axis. In order to further elucidate the involvement of the CXCR4/SDF-1 $\alpha$ axis, the ability of G-CSF to reduce the expression levels of surface CXCR4 protein, total CXCR4 protein and CXCR4 mRNA in HL-60 cells was investigated. Notably, the surface expression of CXCR4 was markedly downregulated in the HL-60 cells pre-treated with G-CSF, AMD3100 or with G-CSF plus AMD3100, and similar results were observed for the total CXCR4 protein using western blotting. Notably, the expression levels of surface CXCR4 protein were markedly more decreased in the AMD3100 group, as compared with the G-CSF group; whereas total CXCR4 protein expression was markedly decreased in the HL-60 cells that had been pre-treated with G-CSF, but not in those pre-treated with AMD3100. In 2011, Spinello et al (32) reported that acute treatment (1-4 h) with $10 \mu \mathrm{g}$ AMD3100 elicited rapid downregulation of surface CXCR4 expression, without any significant modulation of CXCR4 mRNA and total protein expression levels. Therefore, the authors of the present study hypothesized that these differences may be due to differences in the mechanisms used by G-CSF and AMD3100 to downregulate CXCR4 expression. AMD3100 may have decreased CXCR4 surface expression via receptor internalization, whereas G-CSF may have inhibited CXCR4 expression via translational repression. Analysis of the expression levels of CXCR4 mRNA and miR-146a supported this hypothesis. G-CSF significantly upregulated miR-146a expression levels and downregulated CXCR4 mRNA expression levels, whereas AMD3100 did not. To the best of our knowledge, the present study is the first to demonstrate that G-CSF and AMD3100 may utilize different mechanisms in the downregulation of CXCR4 expression and exhibit synergistic anti-leukemia activity in vitro.

In conclusion, the present study demonstrated that G-CSF was able to overcome stromal-mediated drug resistance in the HL-60 cell line by interfering with the CXCR4/SDF-1 $\alpha$ axis, likely via the upregulation of miR-146a expression in order to reduce CXCR4 expression. These results suggested that there may be broader prospects for the clinical application of $\mathrm{G}-\mathrm{CSF}$, as well as its use as a mobilization agent.

\section{Acknowledgements}

The present study was supported by the National Natural Science Foundation of China (grant no. 81270626).

\section{References}

1. Zhu X, Ma Y and Liu D: Novel agents and regiments for acute myeloid leukemia: 2009 ASH annual meeting highlights. J Hematol Oncol 3: 17, 2010.

2. Estey EH: Acute myeloid leukemia: 2012 update on diagnosis, risk stratification and management. Am J Hematol 87: 89-99, 2012.

3. Rowe JM and Tallman MS: How I treat acute myeloid leukemia. Blood 116: 3147-3156, 2010

4. Gregory TK, Wald D, Chen Y, Vermaat JM, Xiong Y and Tse W: Molecular prognostic markers for adult acute myeloid leukemia with normal cytogenetics. J Hematol Oncol 2: 23, 2009.

5. Juliusson G, Antunovic P, Derolf A, Lehmann S, Mollgard L, Stockelberg D, Tidefelt U, Wahlin A and Hoglund M: Age and acute myeloid leukemia: Real world data on decision to treat and outcomes from the Swedish Acute Leukemia Registry. Blood 113: 4179-4187, 2009. 
6. Yamada K, Furusawa S, Saito K, Waga K, Koike T, Arimura H, Aoyagi A, Yamato H, Sakuma H, Tsunogake S, et al: Concurrent use of granulocyte colony-stimulating factor with low-dose cytosine arabinoside and aclarubicin for previously treated acute myelogenous leukemia: A pilot study. Leukemia 9: 10-14, 1995

7. Wei G, Ni W, Chiao JW, Cai Z, Huang H and Liu D: A meta-analysis of CAG (cytarabine, aclarubicin, G-CSF) regimen for the treatment of 1029 patients with acute myeloid leukemia and myelodysplastic syndrome. J Hematol Oncol 4: 46, 2011.

8. Collins RJ, Verschuer LA, Harmon BV, Prentice RL, Pope JH and Kerr JF: Spontaneous programmed death (apoptosis) of B-chronic lymphocytic leukaemia cells following their culture in vitro. Br J Haematol 71: 343-350, 1989.

9. Nwajei F and Konopleva M: The bone marrow microenvironment as niche retreats for hematopoietic and leukemic stem cells. Adv Hematol 2013: 953982, 2013.

10. Mèndez-Ferrer S, Michurina TV, Ferraro F, Mazloom AR, Macarthur BD, Lira SA, Scadden DT, Ma'ayan A, Enikolopov GN and Frenette PS: Mesenchymal and haematopoietic stem cells form a unique bone marrow niche. Nature 466: 829-834, 2010.

11. Caligaris-Cappio F: Role of the microenvironment in chronic lymphocytic leukaemia. Br J Haematol 123: 380-388, 2003.

12. Azab AK, Runnels JM, Pitsillides C, Moreau AS, Azab F, Leleu X, Jia X, Wright R, Ospina B, Carlson AL, et al: CXCR4 inhibitor AMD3100 disrupts the interaction of multiple myeloma cells with the bone marrow microenvironment and enhances their sensitivity to therapy. Blood 113: 4341-4351, 2009.

13. Yin T and Li L: The stem cell niches in bone. J Clin Invest 116 1195-1201, 2006.

14. Zeng Z, Shi YX, Samudio IJ, Wang RY, Ling X, Frolova O, Levis M, Rubin JB, Negrin RR, Estey EH, et al: Targeting the leukemia microenvironment by CXCR4 inhibition overcomes resistance to kinase inhibitors and chemotherapy in AML. Blood 113: 6215-6224, 2009

15. Tavor S, Eisenbach M, Jacob-Hirsch J, Golan T, Petit I, Benzion K, Kay S, Baron S, Amariglio N, Deutsch V, et al: The CXCR4 antagonist AMD3100 impairs survival of human AML cells and induces their differentiation. Leukemia 22: 2151-5158, 2008.

16. Sison EA, Rau RE, McIntyre E, Li L, Small D and Brown P: MLL-rearranged acute lymphoblastic leukaemia stem cell interactions with bone marrow stroma promote survival and therapeutic resistance that can be overcome with CXCR4 antagonism. Br J Haematol 160: 785-797, 2013

17. Busillo JM and Benovic JL: Regulation of CXCR4 signaling. Biochim Biophys Acta 1768, 952-963, 2007.

18. Vianello F, Villanova F, Tisato V, Lymperi S, Ho KK, Gomes AR, Marin D, Bonnet D, Apperley J, Lam EW and Dazzi F: Bone marrow mesenchymal stromal cells non-selectively protect chronic myeloid leukemia cells from imatinib-induced apoptosis via the CXCR4/CXCL12 axis. Haematologica 95: 1081-1089, 2010.

19. Burger JA and Kipps TJ: CXCR4: A key receptor in the crosstalk between tumor cells and their microenvironment. Blood 107: 1761-1767, 2006.

20. He L and Hannon GJ: MicroRNAs: Small RNAs with a big role in gene regulation. Nat Rev Genet 5: 522-531, 2004.

21. Chen Y, Stamatoyannopoulos G and Song CZ: Down-regulation of CXCR4 by inducible small interfering RNA inhibits breast cancer cell invasion in vitro. Cancer Res 63: 4801-4804, 2003.

22. Labbaye C, Spinello I, Quaranta MT, Pelosi E, Pasquini L, Petrucci E, Biffoni M, Nuzzolo ER, Billi M, Foà R, et al: A three-step pathway comprising PLZF/miR-146a/CXCR4 controls megakaryopoiesis. Nat Cell Biol 10: 788-801, 2008.

23. Kim HK, De La Luz Sierra M, Williams CK, Gulino AV and Tosato G: G-CSF down-regulation of CXCR4 expression identified as a mechanism for mobilization of myeloid cells. Blood 108 812-820, 2006
24. De La Luz Sierra M, Gasperini P, McCorimick PJ, Zhu J and Tosato G: Transcription factor Gfi-1 induced by G-CSF is a negative regulator of CXCR4 in myeloid cells. Blood 110 2276-2285, 2007

25. Donahue RE, Jin P, Bonifacino AC, Metzger ME, Ren J, Wang E and Stroncek DF: Plerixafor (AMD3100) and granulocyte colony-stimulating factor (G-CSF) mobilize different CD34+ cell populations based on global gene and microRNA expression signatures. Blood 114: 2530-2541, 2009.

26. Livak KJ and Schmittgen TD: Analysis of relative gene expression data using real-time quantitative PCR and the 2(-Delta Delta C(T)) Method. Methods 25: 402-408, 2001.

27. Förster R, Kremmer E, Schubel A, Breitfeld D, Kleinschmidt A, Nerl C, Bernhardt G and Lipp M: Intracellular and surface expression of the HIV-1 coreceptor CXCR4/fusin on various leukocyte subsets: Rapid internalization and recycling upon activation. J Immunol 160: 1522-1531, 1998.

28. Fernandez HF, Sun Z, Yao X, Litzow MR, Luger SM, Paietta EM, Racevskis J, Dewald GW, Ketterling RP, Bennett JM, et al: Anthracycline dose intensification in acute myeloid leukemia. N Engl J Med 361: 1249-1259, 2009.

29. Bai A, Kojima H, Hori M, Nara N, Komeno T, Hasegawa Y, Ninomiya $H$, Abe T and Nagasawa T: Priming with G-CSF effectively enhances low-dose Ara-C-induced in vivo apoptosis in myeloid leukemia cells. Exp Hematol 27: 259-265, 1999.

30. Tafuri A and Andreeff M: Kinetic rationale for cytokine-induced recruitment of myeloblastic leukemia followed by cycle-specific chemotherapy in vitro. Leukemia 4: 826-834, 1990.

31. Ferrero D, Carlesso N, Pregno P, Gallo E and Pileri A: Self-renewal inhibition of acute myeloid leukemia clonogenic cells by biological inducers of differentiation. Leukemia 6: 100-106, 1992.

32. Spinello I, Quaranta MT, Riccioni R, Riti V, Pasquini L, Boe A, Pelosi E, Vitale A, Foà R, Testa U and Labbaye C: MicroRNA-146a and AMD3100, two ways to control CXCR4 expression in acute myeloid leukemias. Blood Cancer J 1: e26, 2011.

33. Dalton WT Jr, Ahearn MJ, McCredie KB, Freireich EJ, Stass SA and Trujillo JM: HL-60 cell line was derived from a patient with FAB-M2 and not FAB-M3. Blood 71: 242-247, 1988.

34. Hendrix CW, Flexner C, MacFarland RT, Giandomenico C, Fuchs EJ, Redpath E, Bridger G and Henson GW: Pharmacokinetics and safety of AMD-3100, a novel antagonist of the CXCR-4 chemokine receptor, in human volunteers. Antimicrob Agents Chemother 44: 1667-1673, 2000.

35. Stamatopoulos B, Meuleman N, De Bruyn C, Pieters K, Mineur P, Le Roy C, Saint-Georges S, Varin-Blank N, Cymbalista F, Bron D and Lagneaux L: AMD3100 disrupts the cross-talk between chronic lymphocytic leukemia cells and a mesenchymal stromal or nurse-like cell-based microenvironment: Pre-clinical evidence for its association with chronic lymphocytic leukemia treatments. Haematologica 97: 608-615, 2012.

36. Campbell JJ, Qin S, Bacon KB, Mackay CR and Butcher EC: Biology of chemokine and classical chemoattractant receptors: differential requirements for adhesion-triggering versus chemotactic responses in lymphoid cells. J Cell Biol 134: 255-266, 1996.

37. Flomenberg N, Devine SM, DiPersio JF, Liesveld JL, McCarty JM, Rowley SD, Vesole DH, Badel K and Calandra G: The use of AMD3100 plus G-CSF for autologuos hematopoietic progentior cell mobilization is superior to G-CSF alone. Blood 106: 1867-1874, 2005.

38. Winkler IG, Pettit AR, Raggatt LJ, Jacobsen RN, Forristal CE, Barbier V, Nowlan B, Cisterne A, Bendall LJ, Sims NA and Lévesque JP: Hematopoietic stem cell mobilizing agents G-CSF, cyclophosphamide or AMD3100 have distinct mechanisms of action on bone marrow HSC niches and bone formation. Leukemia 26: 1594-1601, 2012. 